\section{Simple, Quick Methods for Detection of 6-Amino-penicillanic Acid}

ONE of the greatest discoveries of the past years in penicillin research was the discovery, isolation ${ }^{1}$ and preparation of 6-amino-penicillanic acid (6-APA) forming the common 'nucleus' of natural penicillins $^{2,8}$, and the successful introduction into therapy of semi-synthetic penicillins produced from it ${ }^{4,5}$. The new semi-synthetic penicillin preparations have already made great progress in the fight against resistant bacteria. The possibility is now laid open for the preparation of penicillins possessing novel effectiveness ${ }^{6}$. The basis for efforts of this kind is 6-APA research, and its quick identification in fermentation liquor and other reaction mixtures.

The biological methods hitherto used (such as paper-chromatography; the chemical transformation of 6-APA into some familiar natural penicillin, or bioassay) even if not complicated, are comparatively lengthy. The following two simple methods have been devised for quick detection of 6-APA :

(1) The essence of the paper-slice method is the cutting of $1 \mathrm{~cm}$.-wide slices from Whatman No. 1 filter-paper in appropriate lengths and washing them first in a solution of 3 per cent sodium bicarbonate, then, after drying, in a solution of 1 per cent phenylacetylchloride in acetone. After drying in air, a solution containing 6-APA is added, drop-wise, to the impregnated paper. If there is 6-APA in the solution, it will be converted to $G$-penicillin on the paper and yield an inhibition zone. This method is appropriate for qualitative determination.

(2) The 6-APA content of fermentation liquid or other solutions is demonstrable also by means of the hole plate method. 6-APA is transformed directly into a natural penicillin within holes in an agar plate inoculated with $B$. subtilis spores. In this manner, the time and effort needed to perform this reaction in a separate test-tube are saved. Fig. 1 illustrates such a demonstration. Each fermentation liquid is measured into two holes. Into one hole (upper row in the figure) a single drop of 3 per cent $\mathrm{NaHCO}_{3}$ solution is added, while into the other hole (lower row in the figure) one drop of 1 per cent phenylacetylchloride in acetone is also added. The plate is then incubated for $5 \mathrm{hr}$. at $37^{\circ} \mathrm{C}$. If there is 6-APA in the fermentation liquid, it is converted to $G$-penicillin and yields an inhibition zone, as can be seen around the holes 1-4 of the lower row in the figure. Around the holes of the upper row is the inhibition zone due to the penicillin remaining in the fermentation fluid after extraction. The fifth hole, where

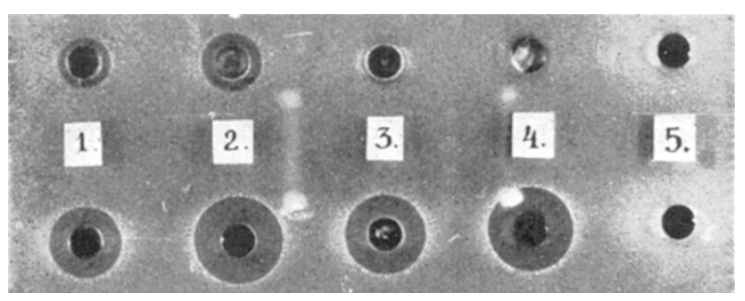
Fig. 1. Detection of 6-APA content of fermentation liquids by
use of the hole plate method.

Test micro-organism: $R$. subtilis, 0.1 ml. of the liquid containing Test micro-organism: $R .8 u b t i t s, 0.1$ mit. of the hquid containing
8-APA (1, 0.04 unit; $2,0.2$ unit; $3,0.06$ unit; $4,0.2$ unit; 5 ,
dist.H,$O$ measured into holes. One drop of 3 per cent $\mathrm{NaHCO}_{3}$, and, on the lower row, an additional drop of 1 per cent phenylacetylchloride in acetone is measured, In the upper row: inhibition zone of remaining natural penicillins. In the lower one: inhibition zone of $G$-penicillin deriving from 6-APA distilled water was introduced, the reagents in the quantity applied do not produce an inhibition zone. This method is applicable for the quantitative, quick detection even of a tenth unit of 6-APA.

Department of Pharmacology,
University Medical School,

J. URI *

'Biogal' Pharmaceutic Factory,

\section{F. Sztaricskai}

Debrecen, Hungary.

* Present address: Department of Biochemistry, London School of Hygiene and Tropical Medicine, Keppel Street, London, W.C.1.

${ }^{1}$ Batchelor, F. R. Doyle, F. P., Nayler, J. H. C., and Rolinson, G. N., Nature, 183, 257 (1959).

"Huang, H. T., Engiish, A. R., Seto, T. A., Shull, G. M., and Sobin, B. A., J. Amer. Chem. Soc., 82, 3790 (1960).

sheehan, J. C., and Henery-Logan, K. R., J. Amer. Chem. Soc., 81, 3089,5838 (1959).

4 Perron, Y. G., Crast, L. B., Gottstein, W. I., Minor, W. F., and Cheney, L. C., Antib. Ann., 107 (1959).

solinson, G. N., Stevens, S., Batchelor, F. R., Cameron Wood, J.,
and Chain, E. B., Lancet, il, 564 (1960). B Rolinson, G. N., and Stevens, S., Brit. Med. J., ii, 191 (1961).

\section{ENTOMOLOGY}

\section{Exchanges of Sodium lons in the Central Nervous System of an Insect (Periplaneta americana L.)}

IN earlier work it was shown that the exchanges of sodium and potassium ions between the hæmolymph and the central nervous system of the cockroach occurred relatively rapidly ${ }^{1}$. It was concluded from these results that the connective tissue layer surrounding the nervous system did not function as a significant diffusion barrier and that in such a dynamic system any selective permeability must be attributed to the underlying cellular layer of the nerve sheath or some associated elements.

An attempt has recently been made to throw some further light on these processes by studying the efflux of sodium ions from isolated abdominal nerve cords and single connectives and ganglia of Periplaneta americana $\mathrm{L}$. In these experiments the rate of loss of sodium-24 was measured by determining the decline in radioactivity of isolated preparations maintained in a flowing physiological solution. The greater part of the efflux of sodium-24 measured in this way was found to approximate to a simple exponential function, both for the whole nerve cord and for the isolated fragments. The presence of $2: 4$-dinitrophenol and cyanide in low concentrations was found to reduce substantially the rate of efflux of sodium measured in these experiments (Fig. 1). Similarly, an absence of potassium ions from the external solution resulted in a slowing down of loss of sodium, although sodium-free solutions appeared to have no appreciable effects on the measured efflux from the nerve cord. Thus it seems that, as for some other cells and tissues ${ }^{2}$, sodium ions are extruded from the central nervous system by a metabolically maintained secretory mechanism which is also associated with the uptake of potassium ions.

In another group of experiments the effect of removing the cellular and fibrous nerve sheath on the rate of sodium extrusion was investigated. It was found that the rate of efflux of sodium-24 from the terminal abdominal ganglion was not appreciably affected by the removal of substantial portions of the perilemma (Fig. 2). It must be concluded, then, that the rate-limiting process measured in these experiments was not the transfer of the ions across 